\title{
Transplante Cardiopulmonar: Quando Indicar?
}

\author{
Cardiopulmonary Transplantation: When to Indicate?
}

Paulo Manuel Pêgo Fernandes, ${ }^{1,2}$ Gabriela Favaro Faria, ${ }^{\circledR}$ Flávio Pola dos Reis, ${ }^{1,2}$ Luís Gustavo Abdalla, ${ }^{1,2}$ José Eduardo Afonso Junior, ${ }^{1}$ Fernando Bacal ${ }^{1,2}$

Hospital Israelita Albert Einstein, ${ }^{1}$ São Paulo, SP - Brasil

Instituto do Coração, Hospital das Clínicas HCFMUSP, Faculdade de Medicina, Universidade de São Paulo, ${ }^{2}$ São Paulo, SP - Brasil

\section{Introdução}

O transplante cardiopulmonar (TCP) teve seu auge no final da década de 1980 e início dos anos 1990, com quase 300 transplantes realizados por ano em todo o mundo. Com os avanços no tratamento da insuficiência cardíaca (IC) e pulmonar, esse número caiu consideravelmente, sendo que, em 2017 foram realizados apenas 62 TCPs em todo o mundo. ${ }^{1,2}$ Existe uma enorme discussão sobre o perfil de pacientes que pode se beneficiar do TCP e o melhor momento em que ele deve ser indicado.

\section{Relato de caso}

Relatamos o caso de uma paciente de 46 anos, com diagnóstico de comunicação atrioventricular (CAV), submetida a correção cirúrgica em 2006, que evoluiu com hipertensão pulmonar secundária em pós-operatório tardio. Apresentava antecedentes pessoais como: acidente vascular cerebral em 2014, com hemiplegia à direita, fibrilação atrial crônica e litíase biliar. Referenciada ao serviço de transplante, a paciente foi incluída em lista de espera para o procedimento após discussão multidisciplinar. Evoluiu com necessidade de internação por dispneia e piora da insuficiência cardíaca classe funcional II para IV (de acordo com a New York Heart Association, NYHA). Uma angiotomografia de tórax mostrou tromboembolismo pulmonar crônico (TEPC) das artérias principais e ramos interlobares. Um ecocardiograma transtorácico mostrou disfunção do ventrículo direito (VD) grave com pressão sistólica da artéria pulmonar (PSAP) de $129 \mathrm{mmHg}$, presença de fluxo bidirecional transeptal, e pelo menos dois orifícios compatíveis com comunicação interatrial $(\mathrm{ClA})$ do tipo ostium secundum, medindo $10 \mathrm{~mm}$ e $8 \mathrm{~mm}$, respectivamente.

A paciente evoluiu com progressão da disfunção cardíaca e piora do quadro clínico (dispneia aos mínimos esforços,

\section{Palavras-chave}

Transplante Coração-Pulmão/tendências; Embolia Pulmonar; Hipertensão Pulmonar; Fibrilação Atrial; Litíase; Acidente Vascular Cerebral.

Correspondência: Paulo Manuel Pêgo Fernandes •

Universidade de São Paulo Instituto do Coração - Cardiopneumologia - Dr.

Eneas de Carvalho Aguiar, 44. CEP 05403-000, São Paulo, SP - Brasil

E-mail: paulo.fernandes@incor.usp.br

Artigo recebido em 26/05/2020, revisado em 20/08/2020, aceito em 09/09/2020

DOI: https://doi.org/10.36660/abc.20200536 cianose central), aumento da pressão sistólica da artéria pulmonar (PSAP $=153 \mathrm{mmHg}$ ) e necessidade de uso de inotrópico (milrinone) contínuo. Foi realizada uma ressonância magnética cardíaca, que identificou retorno da CIA (Figura 1) e TEPC na artéria pulmonar direita (Figura 2).

Após discussão na Câmara Técnica da Central Estadual de Transplantes, a paciente foi priorizada na fila do transplante e, após cinco meses de internação, o TCP foi realizado no Hospital Israelita Albert Einstein (HIAE). O doador era do sexo masculino, 18 anos, e a causa de morte encefálica tinha sido acidente vascular cerebral hemorrágico (AVCH).

A incisão cirúrgica foi feita por bitoracotomia anterior com esternotomia transversal tipo Clamshell, com instalação da circulação extracorpórea (CEC) por meio da canulação na aorta ascendente e drenagem nas veias cavas superior e inferior. Durante a cardiectomia, os nervos frênicos foram identificados bilateralmente e liberados com margens de segurança.

Durante as pneumonectomias, preservou-se a região do nervo laríngeo recorrente para evitar lesões. O implante do bloco cardiopulmonar iniciou-se pelas anastomoses brônquicas, seguidas da aorta e das veias cavas. O tempo de isquemia do enxerto foi 255 minutos e o tempo de CEC, 195 minutos. Após saída da CEC, a paciente foi submetida a tromboelastograma e coagulograma, corrigido conforme resultado com plaquetas, fibrinogênio e complexo protrombínico, além de dois concentrados de hemácias.

A paciente foi admitida na unidade de terapia intensiva em ventilação mecânica, recebendo $0,5 \mathrm{micro} / \mathrm{kg} / \mathrm{min}$ de noradrenalina; 0,06 micro $/ \mathrm{kg} / \mathrm{min}$ de vasopressina; 3,7 micro/ $\mathrm{kg} / \mathrm{min}$ de dobutamina. A profilaxia utilizada foi meropenem e vancomicina e, na indução, metilprednisolona e basiliximabe. Para a imunossupressão, utilizamos Tacrolimus, prednisona e micofenolato.

A paciente foi entubada no segundo dia pós-operatório, com relação $\mathrm{PO}_{2} / \mathrm{FiO}_{2}$ de 400 . Nas primeiras 72 horas após o transplante, a paciente apresentou disfunção primária do enxerto I (DPE), apenas pela alteração radiológica, sem repercussão clínica. Permaneceu na unidade de terapia intensiva por quatro dias e recebeu alta hospitalar no 34 o dia pós-operatório. Atualmente, está em acompanhamento ambulatorial, referindo boa qualidade de vida.

\section{Discussão}

A International Society for Heart and Lung Transplantation (ISHLT) reporta que a maior indicação para o TCP continua sendo a hipertensão pulmonar, devido à hipertensão arterial pulmonar idiopática ou secundária a doenças 


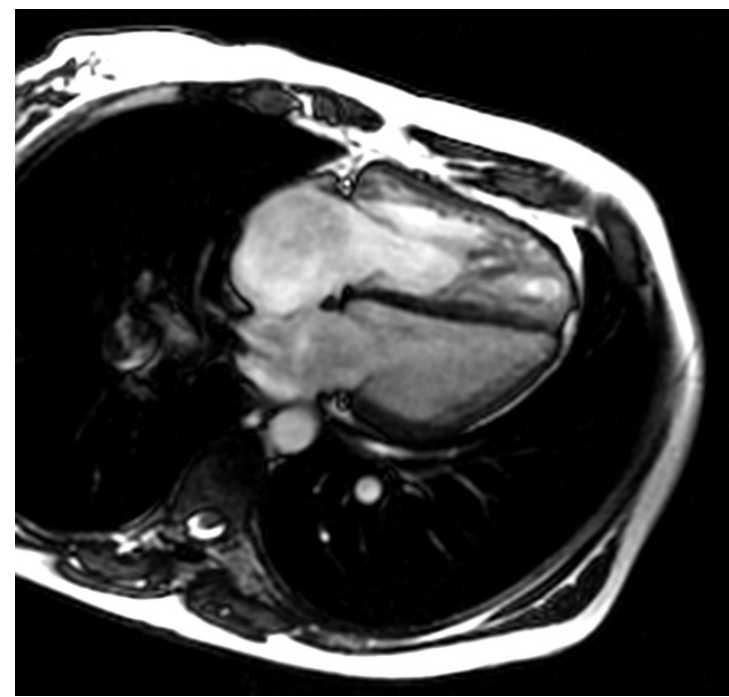

Figura 1 - Ressonância magnética cardíaca mostrando comunicação interatrial.

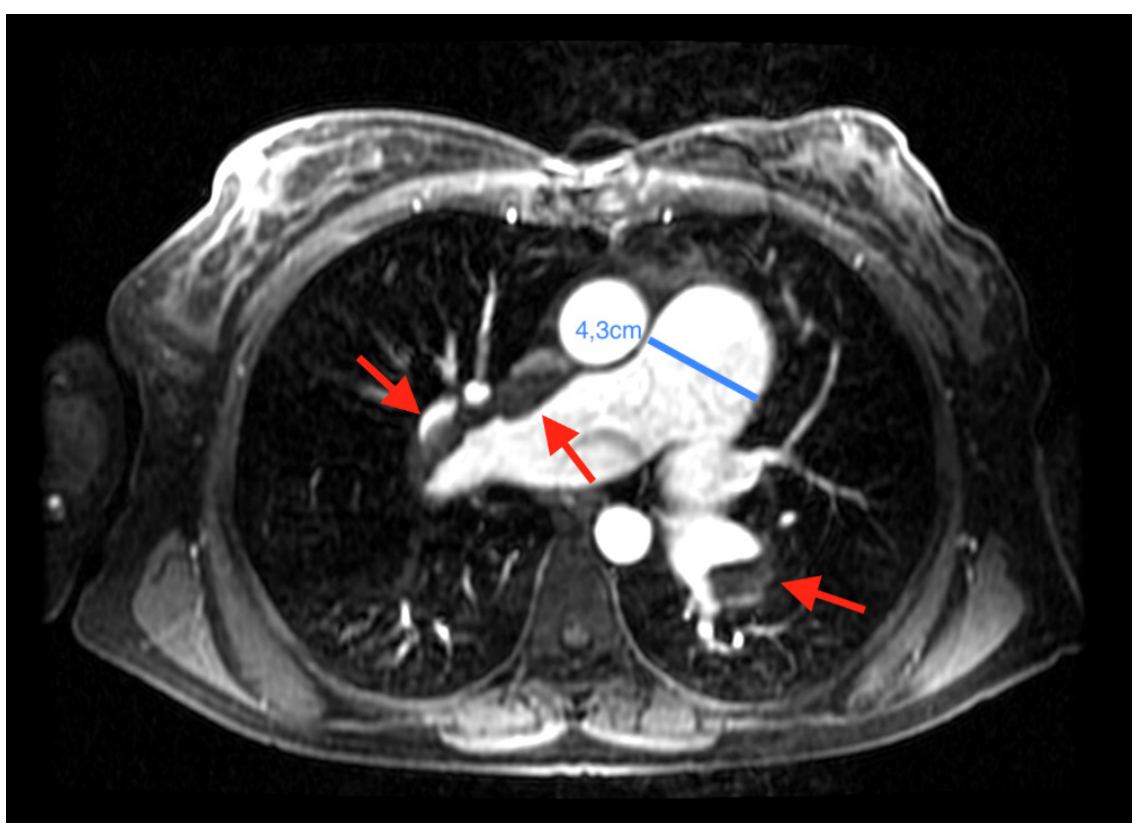

Figura 2 - Ressonância magnética cardiaca evidenciando tromboembolismo na artéria pulmonar direita (seta vermelha) e o aumento do tronco da artéria pulmonar.

cardíacas congênita (como a Sindrome de Eisenmenger), que representa $60 \%$ a $70 \%$ dos transplantes nas últimas três décadas, seguida da Fibrose Cística com 14,9\%., 3,4

A opção pelo transplante isolado de coração e pulmão para os pacientes que antigamente seriam tratados com o TCP, bem como os avanços no tratamento da hipertensão pulmonar refletiu na diminuição do número de TCP realizados.

Os exames pré-operatórios devem ser criteriosamente analisados, pois os pacientes que serão indicados e submetidos ao TCP podem ter comprometimento de outros órgãos, como fígado e rim, além de congestão venosa sistêmica crônica. Outro ponto a ser verificado é se o receptor tem painel imunológico positivo, pois, devido a procedimentos prévios com transfusões, o paciente pode ser sensibilizado. ${ }^{5}$

O manejo pós-operatório do TCP é semelhante ao dos pacientes submetidos ao transplante pulmonar isoladamente. As causas comuns de morte nos primeiros 30 dias são falência 


\section{Carta Científica}

do enxerto, complicações técnicas e infecção. A síndrome de bronquiolite obliterante (BOS) e a disfunção do aloenxerto pulmonar (DEP) continuam sendo as principais causas de mortalidade no primeiro ano. ${ }^{6}$

Há na literatura internacional uma grande discussão sobre a necessidade e a indicação do TCP ou quando recomendar o transplante pulmonar ou cardíaco isoladamente. No Brasil, a $3^{\underline{a}}$ Diretriz Brasileira de Transplante Cardíaco aconselha que, em casos de hipertensão pulmonar fixa, o TCP pode ser considerado. ${ }^{7}$ Porém, alguns pontos devem ser levados em consideração: a anatomia, o agravamento da insuficiência ventricular, hipertensão, condições clínicas e hemodinâmicas do paciente e piora da qualidade de vida, índice cardíaco e disfunção renal. ${ }^{8}$

Uma desvantagem do TCP é o uso de um doador para um paciente, enquanto poderiam ser realizados um transplante cardíaco e pulmonar bilateral ou dois unilaterais, em dois ou três pacientes. ${ }^{9}$

Existem muitos pacientes no Brasil que podem se beneficiar do TCP, seja por cardiopatia congênita ou por Hipertensão Arterial Pulmonar (HAP) de qualquer etiologia, em que o acometimento é severo e irreversível. ${ }^{10,11}$

\section{Conclusão}

O TCP deve ser considerado como opção terapêutica para pacientes cuidadosamente selecionados. O momento de indicação para o transplante, antes que a doença se agrave, é de fundamental importância para atingir bons resultados. O manejo desses pacientes requer cuidado complexo e multidisciplinar. Há uma demanda em parte oculta em nossa população que pode se beneficiar desse tipo de transplante.

\section{Referências}

1. Webb W R, Howard H S. Cardio-pulmonary Transplantation. Surg Forum. 1957;8:313-7.

2. Reitz BA, Wallwork JL, Hunt SA, Pennock JL, Billingham ME, Oyer PE, et al. Heart-Lung Transplantation: Successful Therapy for Patients with Pulmonary Vascular Disease. N Engl J Med. 1982 Mar 11;306(10):557-64.

3. K. Khush K, Cherikh WS, Chambers DC, Goldfarb S, Hayes D, Kucheryavaya AY, et al. The International Thoracic Organ Transplant Registry of the International Society for Heart and Lung Transplantation: Thirty-fifth Adult Heart Transplantation Report-2018; Focus Theme: Multiorgan Transplantation. J Heart Lung Transplant. 2018;37(10):1155-68.

4. Goldfarb SB, Hayes D, Levvey BJ, Cherikh WS, Chambers DC, Khush KK, et al. The International Thoracic Organ Transplant Registry of the International Society for Heart and Lung Transplantation: Twenty-first Pediatric Lung and Heart-Lung Transplantation Report-2018; Focus Theme: Multiorgan Transplantation. J Heart Lung Transplant. 2018;37(10):1196-206.

5. Brouckaert J, Verleden SE, Verbelen T, Coosemans W, Decaluwé H, De Leyn $\mathrm{P}$, et al. Double-lung versus heart-lung transplantation for precapillary pulmonary arterial hypertension: a 24-year single-center retrospective study. Transpl Int.2019;37(10):196-206.

\section{Contribuição dos autores}

Concepção e desenho da pesquisa: Fernandes PMP; Obtenção de dados: Reis FP, Abdalla LG; Redação do manuscrito: Faria GF; Revisão crítica do manuscrito quanto ao conteúdo intelectual importante: Fernandes PMP, Afonso Junior JE, Bacal F.

\section{Potencial conflito de interesses}

Declaro não haver conflito de interesses pertinentes.

\section{Fontes de financiamento}

O presente estudo não teve fontes de financiamento externas.

\section{Vinculação acadêmica}

Não há vinculação deste estudo a programas de pósgraduação.

\section{Aprovação ética e consentimento informado}

Este estudo foi aprovado pelo Comitê de Ética da Sociedade Beneficente Israelita Brasileira Albert Einstein sob o número de protocolo 4.038.465. Todos os procedimentos envolvidos nesse estudo estão de acordo com a Declaração de Helsinki de 1975, atualizada em 2013. O consentimento informado foi obtido de todos os participantes incluídos no estudo.
6. Toyoda Y, Toyoda Y. Heart-lung transplantation: Adult indications and outcomes. J Thorac Dis. 2014;6(8):1138-42.

7. Bacal F, Marcondes-Braga FG, Rohde LEP, Xavier Júnior JL, de Souza Brito F, Moura LZ, et al. 3a Diretriz Brasileira de Transplante Cardíaco. Arq Bras Cardiol. 2018; 111(2):230-89

8. Idrees JJ, Pettersson GB. State of the Art of Combined Heart-Lung Transplantation for Advanced Cardiac and Pulmonary Dysfunction. Vol. 18, Current Cardiol Rep. Current Medicine Group LLC 1; 2016. p. 1-9.

9. Le Pavec J, HascoëtS, Fadel E. Heart-lung transplantation: current indications, prognosis and specific considerations. J Thorac Dis. 2018;26(10:5946-52.

10. Associação Brasileira de Transplantes de Orgãos . (ABTO). Dimensionamento dos Transplantes no Brasil e em cada estado [cited 2020 May 26];1-89. Available from: www.abto.org.br

11. Pêgo-Fernandes PM. Heart-lung transplantation: a necessity. J Bras Pneumol. 2020 May;46(3):e20190273-e20190273. 